M. R. Yaghouti, M. Malzoumati, H. Deilami / TJMCS Vol .4 No.4 (2012) 514-522

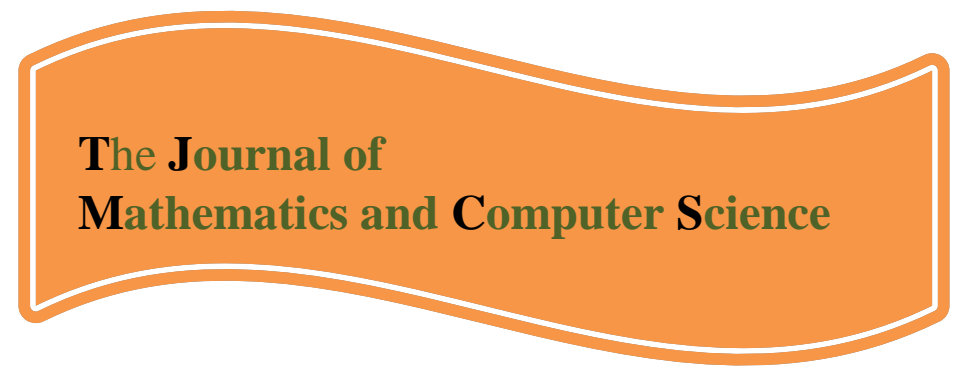

Available online at

\title{
http://www.TIMCS.com
}

The Journal of Mathematics and Computer Science TJMCS Vol .4 No.4 (2012) 514-522

\section{A Numerical Approach of a Family of Smoluchowski's Equations by Use of Adomian Decomposition Method}

\author{
Mohammad Reza Yaghouti \\ The Department of Mathematics Science, University of Guilan, Rasht, Iran \\ yaghouti@guilan.ac.ir \\ Marzie Malzoumati \\ The Department of Mathematics Science, University of Guilan, Rasht, Iran \\ mmalzoumati@gmail.com \\ Haman Deilami \\ The Department of Mathematics Science, University of Guilan, Rasht, Iran, \\ hamandeilami@yahoo.com
}

Received: 10/28/2012, Revised: $11 / 21 / 2012$

Online Publication: 12/21/2012

\section{Abstract}

The Smoluchowski's equation as a partial differential equation models the diffusion and binary coagulation of a large collection of tiny particles. The mass parameter, indexed either by positive integers, or positive real's, corresponds to the discrete or continuous form of the equations. In this article, we try to use the Adomian's decomposition method (ADM) to approximate the solution of the homogeneous Smolochowski's equation with different kernels. Some test problems have been included to show the accuracy of the method.

Keywords: Adomian's decomposition method, the homogeneous Smoluchowski's equation.

\section{Mathematics Subject Classification: $65 Q 10$.}

\section{Introduction.}

It is a common practice in statistical mechanics to formulate a microscopic model with simple dynamical rules in order to study a phenomenon of interest. In a colloid, a population of comparatively massive particles is agitated by the bombardment of much smaller particles in the ambient environment; the motion of the colloidal particles may 
then be modeled by Brownian motion [8,9]. Smoluchowski's equation provides a macroscopic description for the evolution of the cluster densities in a colloid whose particles are prone to binary coagulation. Smoluchowski's equation comes in two flavors: discrete and continuous. In the discrete version, the cluster mass may take values in the set of positive integers, whereas, in the continuous version, the cluster mass take values in $\mathbb{R}^{+}$. Writing $f_{n}(x, t)$ for the density of clusters (or particles) of size $n$ this density evolves according to

$$
\frac{\partial f_{n}(x, t)}{\partial t}=d(n) \Delta f_{n}(x, t)+Q_{+}^{n}(f)(x, t)-Q_{-}^{n}(f)(x, t) ; \quad x \in \mathbb{R}^{d}
$$

where

$$
Q_{+}^{n}(f)(x, t)=\int_{0}^{n} \beta(m, n-m) f_{m} f_{n-m} d m, \quad Q_{-}^{n}(f)(x, t)=2 \int_{0}^{\infty} \beta(m, n) f_{m} f_{n} d m
$$

In which $d(n) \Delta f_{n}(x, t)$ and $Q_{+}^{n}(f)(x, t)-Q_{-}^{n}(f)(x, t)$ are respectively diffusion and coagulation parts in the case of the continuous Smoluchowski's equation and $\beta(m, n)$ is considered as a function of the parameters $\alpha(m, n)$ (the microscopic coagulation rate), $d(m)$ and $d(n)$. In the discrete case, the integrations given in (1) are replaced with summations. In [8] and [9], the discrete Smoluchowski's equation is derived as a microscopic model of coagulating Brownian particles. In this paper we consider the continuous homogenous Smoluchowski's equation. The main purpose of this study is to approximate the solution of continuous homogenous Smoluchowski's equation in which the main technical tool is the Adomian's decomposition method. To our knowledge the problem, so far, has not been considered via ADM and other methods have been performed for only constant kernels [13]. However, the issue of more complicated kernels, which so far have been remained unsolved, will be investigated in our future studies. Let us consider the homogenous Smoluchowski's equation without diffusion part:

$\frac{\partial}{\partial t} f(x, t)=\frac{1}{2} N_{1}(f)(x, t)-N_{2}(f)(x, t)$

where

$N_{1}(f)(x, t)=\int_{0}^{x} k(x-y, y) f(x-y, t) f(y, t) d y$

and

$N_{2}(f)(x, t)=\int_{0}^{\infty} k(x, y) f(x, t) f(y, t) d y$

where $N_{1}(f)$ and $N_{2}(f)$ are nonlinear parts in view of ADM. In the next section, we show how ADM works well.

\section{The Decomposition Method [3]}

Eq. (1) may be written in the operator form:

$L f=N_{1}(f)-N_{2}(f), \quad f(x, 0)=f(x)$

and the differential operator $L$ is

$L=\frac{\partial}{\partial t}($.

The inverse operator $L^{-1}$ is an integral operator given by

$L^{-1}()=.\int_{0}^{t}() d$.

Applying $L^{-1}$ upon both sides of (2) and using the initial condition, we find 
$f(x, t)=f(x, 0)+L^{-1}\left(\frac{1}{2} N_{1}(f)-N_{2}(f)\right)$

According to the Adomian's decomposition method the unknown function $f(x, t)$ can be written as

$f(x, t)=\sum_{n=0}^{\infty} f_{n}(x, t)$

Substituting (2) into the functional equation (3) yields

$\sum_{n=0}^{\infty} f_{n}(x, t)=f(x)+L^{-1}\left(\frac{1}{2} \sum_{n=0}^{\infty} A_{n}-\sum_{n=0}^{\infty} B_{n}\right)$

where $f_{0}, f_{1}, \ldots, f_{n}$ are Adomian's polynomials and the components $A_{n}, B_{n}$ 's will be determined recurrently as:

$A_{n}=\frac{1}{n !} \frac{d^{n}}{d \lambda^{n}}\left[N_{1}\left(\sum_{i=0}^{\infty} \lambda^{i} f_{i}\right)\right]_{\lambda=0}, \quad B_{n}=\frac{1}{n !} \frac{d^{n}}{d \lambda^{n}}\left[N_{2}\left(\sum_{i=0}^{\infty} \lambda^{i} f_{i}\right)\right]_{\lambda=0} ; n=0,1,2, \ldots$

It is well known that these polynomials can be constructed for all classes of nonlinearity in view of the algorithms set by Adomian[3] and recently developed by different alternative approaches

$[15,14]$. Thus, we have

$f_{0}(x, t)=f(x, 0), f_{n+1}(x, t)=L^{-1}\left(\frac{1}{2} A_{n}-B_{n}\right) ; n=0,1,2, \ldots$

Note that the first few components of $f_{n}(x, t)$ follow immediately upon setting [1]:

$f_{0}(x, t)=f(x)$,

$f_{1}(x, t)=L^{-1}\left(\frac{1}{2} A_{0}-B_{0}\right)$,

$f_{2}(x, t)=L^{-1}\left(\frac{1}{2} A_{1}-B_{1}\right), \ldots$

It is, in principle, possible to calculate more components in the decomposition series to enhance the approximation and recursively determine more terms of the series $\sum_{n=0}^{\infty} f_{n}(x, t)$; hence the solution $f(x, t)$ is readily obtained in a series form as:

$\varphi_{n}(x, t)=\sum_{k=0}^{\infty} f_{k}(x, t) ; n \geq 0$

where $\lim _{n \rightarrow \infty} \phi_{n}=f(x, t)[8,9]$.

Moreover, the decomposition method series (4) solutions generally converge very rapidly in real physical problems [4]. The convergence of decomposition series have investigated by several authors [5, 7, and 11], in which they have obtained some results about the speed of convergence of ADM applicable in linear and nonlinear functional equations.

\section{Applications}

In this section, the method is applied to some numerical examples.

Example 3.1. Let us examine the homogenous Smoluchowski's equation subject to the initial condition [7] $f(x, 0)=e^{-x}$

(5)

Now, consider the equation (3) subject to initial condition (5) with $k(x, y)=x * y$ and $f(x)=e^{-x}$. In Figures 1, we demonstrate the approximate solutions with different ranges of $x$ and $t$.

Applying the inverse operator $L^{-1}$ on both sides of (2) and using the decomposition series, one gets

$\sum_{n=0}^{\infty} f_{n}(x, t)=e^{-x}+\frac{1}{2} L^{-1}\left[\int_{0}^{x}(x-y) y\left(\sum_{n=0}^{\infty} \lambda^{n} f_{n}(x-y, t)\right)\left(\sum_{n=0}^{\infty} \lambda^{n} f_{n}(y, t)\right) d y-\right.$

$$
\left.-\int_{0}^{\infty} x y\left(\sum_{n=0}^{\infty} \lambda^{n} f_{n}(x, t)\right)\left(\sum_{n=0}^{\infty} \lambda^{n} f_{n}(y, t)\right) d y\right]
$$


Proceeding as before, the Adomian's decomposition method [3, 2, and 6] gives the recurrence relations:

$f_{1}(x, t)=\frac{1}{12} x^{3} e^{-x} t-x e^{-x} t, \ldots$

$$
f_{0}(x, t)=e^{-x}
$$

(7)

where $A_{n}, B_{n}$ 's are Adomian's polynomials that represent the nonlinear terms, given by $A_{0}=\int_{0}^{x}(x-y) y e^{-x+y} e^{-y} d y$,

$A_{1}=\frac{1}{1 !} \frac{d}{d \lambda} \int_{0}^{x}(x-y) y\left(e^{-x+y}+\lambda\left(\frac{1}{2}(x-y) e^{-(x-y)} t-e^{-(x-y)} t\right)\right)\left(e^{-y}+\lambda\left(\frac{1}{2} y e^{-y} t-\right.\right.$

$\left.\left.e^{-y} t\right)\right) d y, \ldots$

$B_{0}=\int_{0}^{\infty} x y e^{-x} e^{-y} d y$,

$B_{1}=\frac{1}{1 !} \frac{d}{d \lambda} \int_{0}^{\infty} x y\left(e^{-x}+\lambda\left(\frac{1}{2} x e^{-x} t-e^{-x} t\right)\right)\left(e^{-y}+\lambda\left(\frac{1}{2} y e^{-y} t-e^{-y} t\right)\right) d y, \ldots$

(8)

Now in view of (5-8), the solution in series form is

$f(x, y)=e^{-x}+\frac{1}{12} x^{3} e^{-x} t-x e^{-x} t+\frac{1}{360} x^{4} t^{2} e^{-x}\left(x^{2}-30\right)-\frac{1}{12} t^{2} e^{-x} x^{2}\left(x^{2}-12\right)+\cdots$

In Figures 1, we demonstrate the approximate solutions with different ranges of $x$ and $t$.

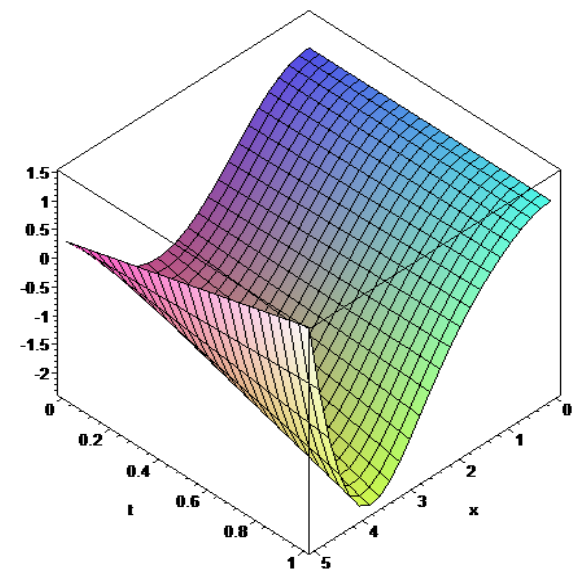

Fig 1.The approximation solution of Example 3.1.

Example 3.2. Let us examine the homogenous Smoluchowski's equation subject to the initial condition

$f(x, 0)=e^{-x}$ with kernel $k(x, y)=x+y$. After calculations, we have

$$
\begin{gathered}
f(x, t)=e^{-x}+\frac{1}{2} x^{2} e^{-x} t-e^{-x}(x+1) t+\frac{1}{6} x^{2} t^{2} e^{-x}\left(x^{2}-3 x-6\right) \\
-\frac{1}{2} t^{2} e^{-x}\left(x^{3}-x^{2}-6 x-2\right)+\cdots
\end{gathered}
$$


M. R. Yaghouti, M. Malzoumati, H. Deilami / TJMCS Vol .4 No.4 (2012) 514-522

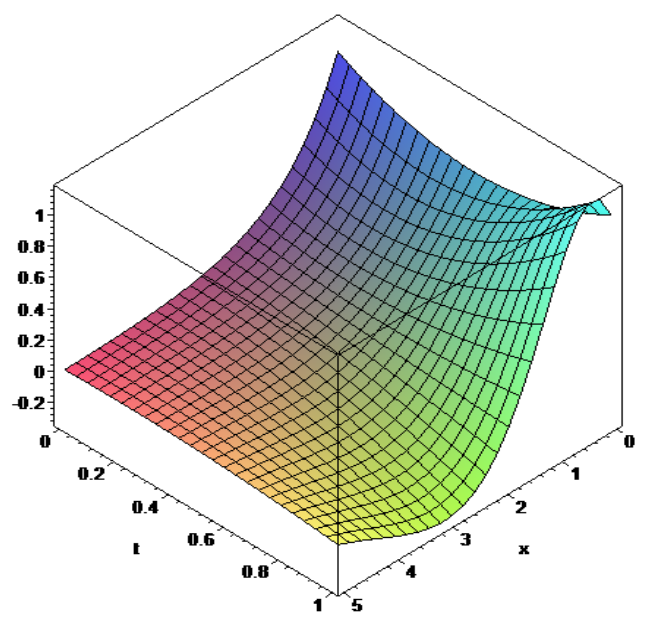

Fig 2. The approximation solution of Example 3.2.

Example 3.3. Let us examine the homogenous Smoluchowski's equation subject to the initial condition

$f(x, 0)=e^{-x}$ with kernel $k(x, y)=x-y$. After calculations, we have $f(x, y)=e^{-x}-e^{-x}(x-1) t+x(x-2) e^{-x} t^{2}+\cdots$

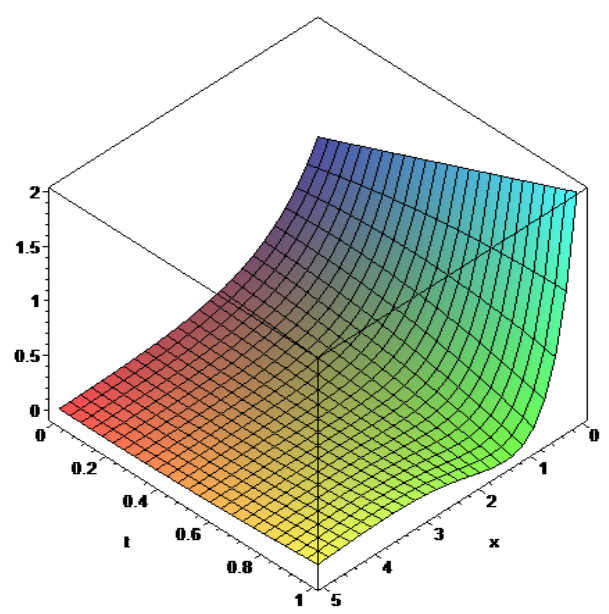

Fig 3. The approximation solution of Example 3.3.

Example 3.4. Let us examine the homogenous Smoluchowski's equation subject to the initial condition

$f(x, 0)=e^{-x}$ with kernel $k(x, y)=\frac{x}{y}$. After calculations, we have $f(x, y)=e^{-x}$. 


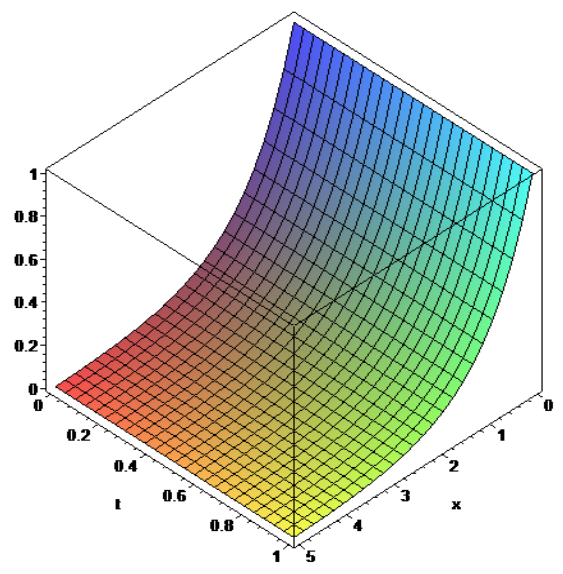

Fig 4. The approximation solution of Example 3.4.

Example 3.5. Let us examine the homogenous Smoluchowski's equation subject to the initial condition

$f(x, 0)=\sin (x)$ with kernel $k(x, y)=x * y$. After calculations, we have $f(x, y)=\sin (x)+\frac{1}{8} \sin (x) t-\frac{1}{8} x \cos (x) t-\frac{1}{24} \cos (x) x^{3} t$.

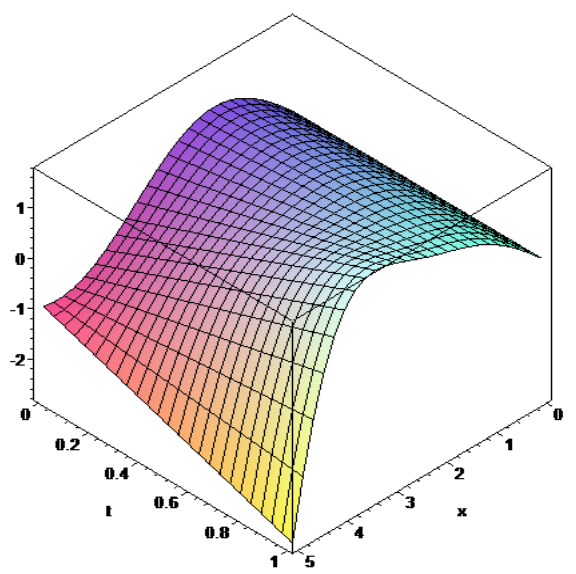

Fig 5. The approximation solution of Example 3.5.

Example 3.6. Let us examine the homogenous Smoluchowski's equation subject to the initial condition

$f(x, 0)=\cos (x)$ with kernel $k(x, y)=x * y$. After calculations, we have $f(x, y)=\cos (x)+\frac{1}{8} \sin (x) t-\frac{1}{8} x \cos (x) t+\frac{1}{24} \cos (x) x^{3} t$. 


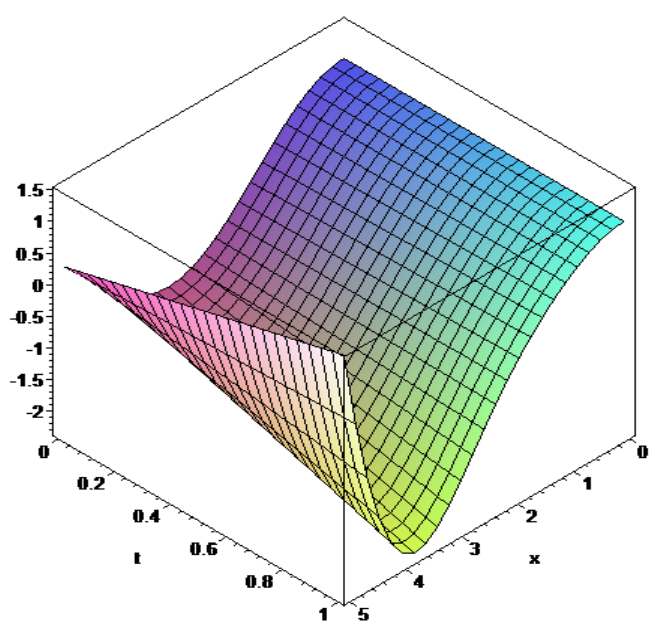

Fig 6. The approximation solution of Example 3.6.

Example 3.7. Let us examine the homogenous Smoluchowski's equation subject to the initial condition

$f(x, 0)=\cos (x)$ with kernel $k(x, y)=x+y$. After calculations, we have $f(x, y)=\cos (x)+\frac{1}{4} x \sin (x) t+\frac{1}{4} \cos (x) x^{2} t$.

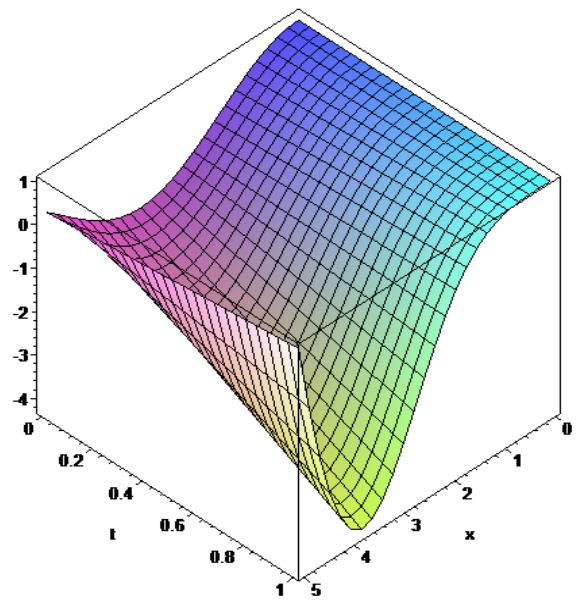

Fig 7. The approximation solution of Example 3.7.

Example 3.8. Let us examine the homogenous Smoluchowski's equation subject to the initial condition

$f(x, 0)=\sin (x)$ with kernel $k(x, y)=x+y$. After calculations, we have $f(x, y)=\sin (x)+\frac{1}{4} x \sin (x) t-\frac{1}{4} \cos (x) x^{2} t$. 


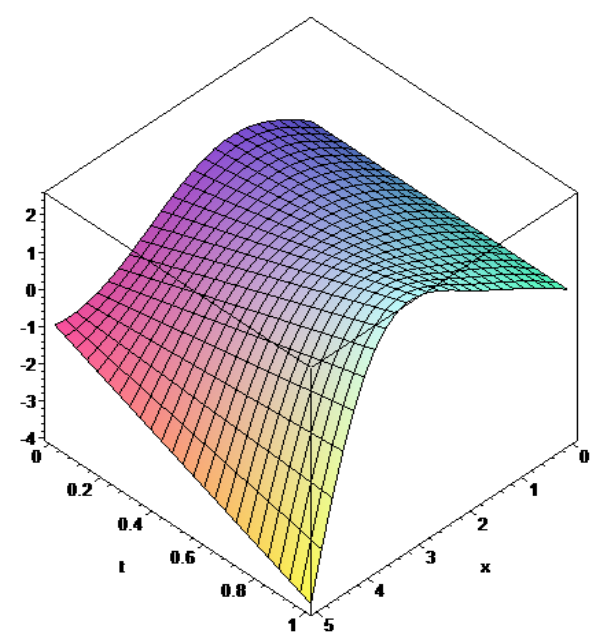

Fig 8. The approximation solution of Example 3.8.

\section{Conclusions}

In this paper, we presented a numerical scheme for solving the continuous homogenous Smoluchowski's equation with different kernels involving at most two variables $x$ and $y$. We have approximated $f(x, t)$ by the Adomian's polynomials. Numerical results show high accuracy of the method as [10].

Acknowledgement. We would like to express our gratitude to all those who gave us the possibility to complete this paper.

\section{References.}

[1] Adomian G. A review of the decomposition method in applied mathematics. J. Math. Anal. Appl. 135, 2(1998),501-544.

[2] Adomian G. Solving frontier problems of physics: the decomposition method. Boston (MA): Kluwer Academic Publishers, (1994).

[3] J. Biazar, S. M. Shafiof. A simple algorithm for calculating Adomian polynomials. Int. J. Contemp Math. Sciences, 2, 20(2007), 975-982.

[4] Y. Cherruault, G. Adomian, Decomposition methods: a new proof of convergence, Math. Comput. Modeling 18 (1993) 103-106.

[5] Y. Cherruault, Convergence of Adomian's method, Kybernetics 18 (1989) 31-38.

[6] Dogan Kaya, Ibrahim E. Inan. A convergence analysis of the ADM and an application. Applied Mathematics and Computation 161, 3(2005), 1015-1025.

[7] F. Filbet, P. Laurencot. Numerical Simulation of Smoluchowski's Coagulation Equation. SIAM J. Sci. Comput. 25, 6(2004), 2004-2008.

[8] A. Hammond, F. Rezakhanlou. The kinetic limit of a system of coagulating Brownian particles. Archive for Relation Mechanics and Analysis, 185, 1. (2007), 1-67. 
M. R. Yaghouti, M. Malzoumati, H. Deilami / TJMCS Vol .4 No.4 (2012) 514-522

[9] A. Hammond, F. Rezakhanlou. Kinetic limit for a system of coagulating planar Brownian particles. J. Stat. Phys. 123(2006), 997-1040.

[10] H. Heidarzadeh, M. Mashinchi Joubari, R. Asghari. Application of Adomian Decomposition Method to Nonlinear Heat Transfer Equation. J. Math. Comp. Sci. 4, 3(2012) 436-447.

[11] Y.C. Jiao, Y.Yamamoto, C.Dong, Y.Hao, An after treatment technique for improving the accuracy of Adomian's decomposition method, Comp. Math. Appl. 43(2000), 783-798.

[12] F. Leyvraz and H.R Tschudi, Singularities in the kinetics of coagulation processes, J. Phys. A. 14(1981), 3389-3405.

[13] M. Ranjbar, H. Adibi, M. Lakestani. Numerical solution of nonlinear ordinary differential equations using Flatlet oblique multiwavelets. Commun. Math. Phys. 296(2010), 769-826.

[14] Wazwaz AM. The decomposition for approximate solution of the Goursat problem. Boston (MA): Kluwer Academic Publishers, (1994).

[15] Wazwaz AM. A reliable modification of Adomian decomposition method. Appl. Math. Comput. 102(1999), 77-86.

[16] Wazwaz AM. A new algorithm for calculating Adomian polynomials for nonlinear operators. Appl. Math. Comput.111(2000), 53-69. 